\title{
Identification of a Minimal 3-Transcript Signature to Differentiate Viral from Bacterial Infection from Best Genome-Wide Host RNA Biomarkers: A Multi-Cohort Analysis
}

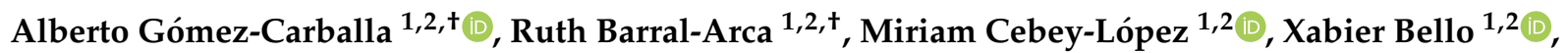 \\ Jacobo Pardo-Seco ${ }^{1,2}$, Federico Martinón-Torres ${ }^{2,3} \mathbb{D}$ and Antonio Salas ${ }^{3,4, *,+}+\mathbb{D}$
}

1 GenPoB Research Group, Instituto de Investigación Sanitaria (IDIS), Hospital Clínico Universitario de Santiago (SERGAS), 15706 Galicia, Spain; Alberto.Gomez.Carballa@sergas.es (A.G.-C.); barralarcaruth@gmail.com (R.B.-A.); Miriam.Cebey.Lopez@sergas.es (M.C.-L.); xbello@gmail.com (X.B.); Jacobo.Jose.Pardo.Seco@sergas.es (J.P.-S.)

2 Genetics, Vaccines and Infections Research Group (GENVIP), Instituto de Investigación Sanitaria de Santiago de Compostela, 15706 Galicia, Spain; federico.martinon.torres@sergas.es

3 Translational Pediatrics and Infectious Diseases, Department of Pediatrics, Hospital Clínico Universitario de Santiago de Compostela, 15706 Galicia, Spain

4 Unidade de Xenética, Instituto de Ciencias Forenses, Facultade de Medicina, Universidade de Santiago de Compostela, 15706 Galicia, Spain

check for updates

Citation: Gómez-Carballa, A.; Barral-Arca, R.; Cebey-López, M.; Bello, X.; Pardo-Seco, J.; Martinón-Torres, F.; Salas, A. Identification of a Minimal 3-Transcript Signature to Differentiate Viral from Bacterial Infection from Best Genome-Wide Host RNA Biomarkers: A Multi-Cohort Analysis. Int. J. Mol. Sci. 2021, 22, 3148. https://doi.org/10.3390/ijms 22063148

Academic Editors: Ancha Baranova and Michael T. Chin

Received: 26 January 2021

Accepted: 15 March 2021

Published: 19 March 2021

Publisher's Note: MDPI stays neutral with regard to jurisdictional claims in published maps and institutional affiliations.

Copyright: (c) 2021 by the authors. Licensee MDPI, Basel, Switzerland. This article is an open access article distributed under the terms and conditions of the Creative Commons Attribution (CC BY) license (https:// creativecommons.org/licenses/by/ $4.0 /)$.
* Correspondence: antonio.salas@usc.es

+ These authors contributed equally to this work.
Abstract: The fight against the spread of antibiotic resistance is one of the most important challenges facing health systems worldwide. Given the limitations of current diagnostic methods, the development of fast and accurate tests for the diagnosis of viral and bacterial infections would improve patient management and treatment, as well as contribute to reducing antibiotic misuse in clinical settings. In this scenario, analysis of host transcriptomics constitutes a promising target to develop new diagnostic tests based on the host-specific response to infections. We carried out a multi-cohort meta-analysis of blood transcriptomic data available in public databases, including 11 different studies and 1209 samples from virus- $(n=695)$ and bacteria- $(n=514)$ infected patients. We applied a Parallel Regularized Regression Model Search (PReMS) on a set of previously reported genes that distinguished viral from bacterial infection to find a minimum gene expression bio-signature. This strategy allowed us to detect three genes, namely BAFT, ISG15 and DNMT1, that clearly differentiate groups of infection with high accuracy (training set: area under the curve (AUC) 0.86 (sensitivity: 0.81; specificity: 0.87); testing set: AUC 0.87 (sensitivity: 0.82; specificity: 0.86)). BAFT and ISG15 are involved in processes related to immune response, while DNMT1 is related to the preservation of methylation patterns, and its expression is modulated by pathogen infections. We successfully tested this three-transcript signature in the 11 independent studies, demonstrating its high performance under different scenarios. The main advantage of this three-gene signature is the low number of genes needed to differentiate both groups of patient categories.

Keywords: RNA; RNAseq; microarrays; transcriptome; transcriptomic biomarkers; RNA signature; multi-cohort; viral infection; bacterial infection; machine learning

\section{Introduction}

According to the World Health Organization (WHO), infectious diseases are still among the major causes of child mortality and are responsible for many medical visits and hospitalizations around the globe [1]. Until recently, it was commonly considered that most severe infections were caused by bacterial pathogens but, during the last decade, increasing evidence shows viral infections as also being responsible for significant morbidity and mortality in children [2]. 
Distinguishing between viral and bacterial infections remains a challenge, since the established bacterial detection methods, such as bacterial culture, can take a few days and even result in false negatives when the infection is located in non-accessible sites [3], or the sample is obtained after an antibiotic treatment [4]. Therefore, out of fear of not diagnosing and properly treating a potentially life-threatening bacterial infection, most clinicians decide to empirically administer antibiotics as a preventive tool while awaiting the bacterial culture test results [4,5]. Consequently, numerous viral infections are erroneously treated with antibiotics, contributing to the appearance of antibiotic-resistant bacteria [4,6]. Antibiotics have contributed to longer and healthier lives, but, as stated by the World Health Organization (WHO), their overuse, together with the absence of current-generation antimicrobial drugs, is enabling common infections and minor injuries to become fatal again.

The development of polymerase chain reaction (PCR)-based molecular assays has noticeably increased the capability to accurately diagnose old and emerging viral infections [7], and also the interrogation of multiple viruses in a single test [8]. Unfortunately, molecular assays have been less efficient in detecting bacterial infections, especially those caused by invasive infections [9]. Furthermore, because these tests point to the presence of nucleic acids, they might not identify the primary causative agent. Therefore, the detected pathogen could no longer be viable, and its presence may simply respond to a recent but unrelated illness [9], or even to an asymptomatic colonization.

In this context, the development of new diagnostic tools is one of the most important challenges of current public healthcare. They will play a central role in the fight against the emergence of bacterial resistance through precise and fast diagnosis, as well as facilitating the correct treatment of bacterial and viral infections.

The human transcriptome is a dynamic layer of information that changes according to cell types and organism conditions. Thus, host transcriptomics approaches not only hold the potential to shed light on the molecular pathogenesis of infectious diseases, but they may also enable the development of new diagnostic approaches based on the host gene expression response to specific pathogens [10,11]. Several host transcriptomic signatures in response to different infections were published in the last decade [4,12-17], but many of them were only focused on the specific pathogen and/or conditions studied, and usually in patients with the same age range or population background. As such, a multi-cohort analysis using publicly available data from different studies can help find common transcriptomic signatures, masking those expression patterns potentially related to specific pathogens, conditions, ages or genetic backgrounds, hence making the translation of these signatures to a generic test and its implementation in the clinical routine more straightforward $[5,18-20]$.

In the present study, we explored host blood gene expression response to different infections to detect key transcriptomic changes related to viral or bacterial pathogens from a multi-cohort perspective. For this purpose, we downloaded 1209 transcriptomic sample profiles from public databases that correspond to 11 different gene expression studies from both microarray and RNA-seq data, containing bacteria- and virus-infected patients from different genetic population backgrounds and ages. We performed a multi-signature metaanalysis of the gene signatures that have been reported in these studies as potentially able to distinguish viral or bacterial infections. Through a machine learning approach, we were able to capture the best minimum transcriptomic signature among these gene candidates.

\section{Results}

To find the best candidates for a specific transcriptomic signature to distinguish viral from bacterial infections, we first combined the 11 different gene expression datasets including a total of 1209 samples (695 samples from viral infections and 514 samples from bacterial infections; Table 1; Table S1), obtaining 3025 common genes between them. Subsequently, we checked for the presence of the 163 different genes that have previously been published in these 11 studies as signature genes with the potential to differentiate 
between viral and bacterial conditions (Table S2) in the 3025 common genes (note that only a few of the 11 articles explored transcript signatures with the capability to separate groups of infection). As a result, 64 out of this initial list of 163 genes could be included in the meta-analysis gene set.

Table 1. Samples included in the meta-analysis (GEO: Gene Expression Omnibus). Platform: lllumina (I), Affymetrix (A); MA: microarray; Cohort: children (C); Adult (A); Source: whole blood (WB), peripheral blood mononuclear cell (PBMCs).

\begin{tabular}{|c|c|c|c|c|c|c|}
\hline GEO ID & $n$ (Virus) & $n$ (Bacteria) & $\begin{array}{c}\text { Platform } \\
\text { Description }\end{array}$ & Cohort & Source & Reference \\
\hline GSE69529 & 80 & 140 & $\begin{array}{l}\text { HiSeq } 2500 \text { (I); } \\
\text { RNA-seq }\end{array}$ & $\mathrm{C}$ & WB & [21] \\
\hline GSE64456 & 190 & 89 & HT12 V4 (I); MA & $\mathrm{C}$ & WB & [19] \\
\hline GSE72829 & 92 & 52 & HT12 V3 (I); MA & $\mathrm{C}$ & WB & [4] \\
\hline GSE6269 & 8 & 16 & $\begin{array}{l}\text { HG U133A Array } \\
\text { (A); MA }\end{array}$ & $\mathrm{C}$ & PBMCs & [22] \\
\hline GSE20346 & 19 & 26 & HT-12 V3 (I); MA & $\mathrm{A}$ & WB & [23] \\
\hline GSE40012 & 39 & 61 & HT-12 V3 (I); MA & $\mathrm{A}$ & WB & [24] \\
\hline GSE40396 & 35 & 8 & HT-12 V4 (I); MA & $\mathrm{C}$ & WB & [25] \\
\hline GSE42026 & 41 & 18 & HT-12 V3 (I); MA & $\mathrm{C}$ & WB & [26] \\
\hline GSE25504 & 3 & 9 & $\begin{array}{c}\text { HG U133 Plus } 2.0 \\
\text { Array (A); MA }\end{array}$ & $\mathrm{C}$ & WB & [27] \\
\hline GSE60244 & 71 & 22 & HT-12 V4 (I); MA & $\mathrm{A}$ & WB & [28] \\
\hline GSE63990 & 117 & 73 & $\begin{array}{c}\text { HG U133 Plus } 2.0 \\
\text { Array (I); MA }\end{array}$ & $\mathrm{A} / \mathrm{C}$ & WB & [29] \\
\hline Totals & 695 & 514 & & & & \\
\hline
\end{tabular}

We performed an over-representation analysis with these 64 candidate genes (Table S2) using both Gene Ontology (GO) and Reactome as the reference pathway database. GO analysis pointed to an implication of these genes in immune response processes ( $p$-adjusted: $\left.3.24 \times 10^{-9}\right)$ mainly driven by the interferon I signaling pathway $\left(1.26 \times 10^{-8}\right)$, the cytokine-mediated signaling pathway ( $p$-adjusted: $2.23 \times 10^{-8}$ ), neutrophil degranulation ( $p$-adjusted: $1.34 \times 10^{-7}$ ), innate immune response ( $p$-adjusted: $\left.2.58 \times 10^{-7}\right)$ and other biological processes related to mechanisms of defense against viral infection ( $p$-adjusted: $9.68 \times 10^{-7)}$ such as negative regulation of viral replication or cell cycle (Figure S1; Table S3). Similar results were achieved when carrying out the over-representation analysis with the Reactome database as the reference: interferon alpha/beta signaling ( $p$-adjusted: $\left.8.74 \times 10^{-9}\right)$, neutrophil degranulation ( $p$-adjusted: $2.15 \times 10^{-6}$ ) innate immune system ( $p$-adjusted: $1.88 \times 10^{-4}$ ) and cytokine signaling in the immune system ( $p$-adjusted: $\left.2.94 \times 10^{-6}\right)($ Figure S2; Table S3). Some of the candidate genes are involved in the IL9 signaling pathway (statistically significative in both over-representation analyses; Table S3).

Among these 64 candidate genes (Table S2), we searched for the minimum transcriptome signature that allows to discriminate between viral and bacterial infections using the optimal gene model size according to the Parallel Regularized Regression Model Search (PReMS) algorithm. To study the expression patterns of these candidate genes in our multi-cohort database, we followed a cross-validation strategy that randomly divides the whole dataset into a training (75\% of the samples) and a test set (remaining $25 \%$ of the samples) both including bacteria- and virus-infected samples. First, we carried out an exploratory analysis on the training set using all candidate genes in the model to assess how the predictive log-likelihood changes with the number of genes included in the signature (Figure S3a). We found that the optimal model was composed of 14 genes (Figure S3b) that clearly separate viral from bacterial infections (Figure 1A) in both the training and the 
test set $\left(p\right.$-value $\left.<2.22 \times 10^{-16}\right)$. We also computed the area under the curve (AUC) of the 14-transcript signature in the training and test cohorts, obtaining values of 0.91 (95\%CI: 0.89-0.91) for the training cohort and 0.87 (95\%CI: 0.83-0.92) for the test cohort (Figure 1B).

A

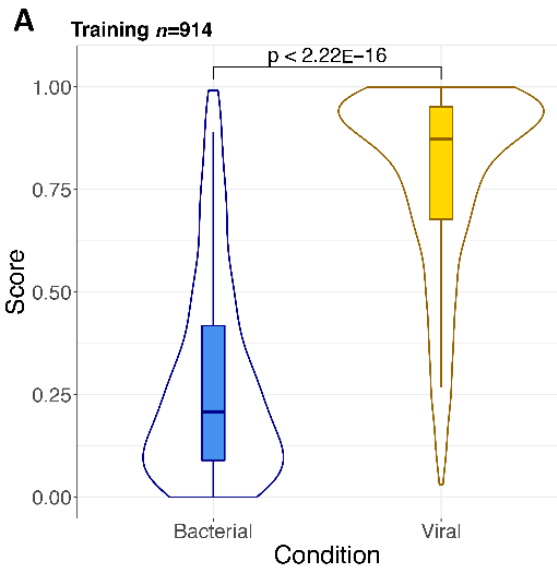

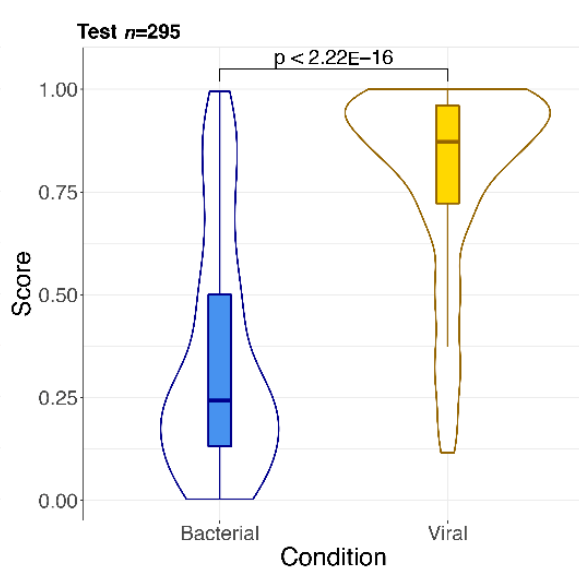

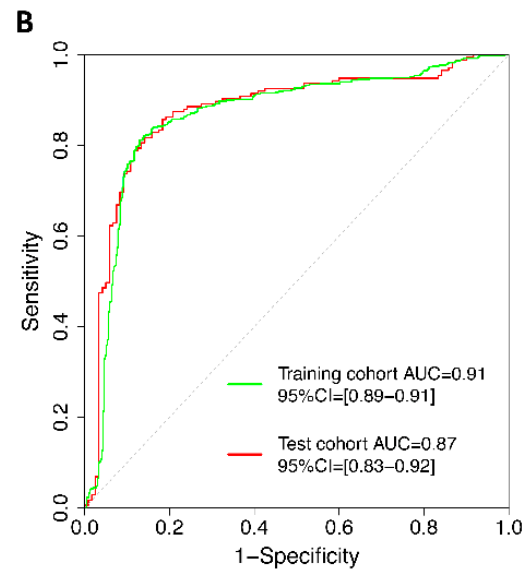

Figure 1. Signature performance based on the 14 genes in the training and test sets. (A) Violin and boxplots of the predicted values from the posterior mean. (B) Receiver operating characteristic (ROC) curves showing the area under the curve (AUC) and $95 \%$ confidence intervals (CIs).

We analyzed in more detail the predictive log-likelihood (Figure S3b) calculated from the training cohort after applying the machine learning algorithm to strike a balance between the size and the accuracy of the gene expression signature. We found that the minimum signature of three genes keeps a predictive value that is only slightly lower compared with the 14-transcript signature; in other words, the addition of genes to the three-transcript model adds very little to the overall predictive value. The minimal signature is composed of genes BATF (Basic Leucine Zipper ATF-Like Transcription Factor), ISG15 (ISG15 Ubiquitin Like Modifier) and DNMT1 (DNA Methyltransferase 1). This signature differentiated bacterial from viral infections with high accuracy (Figure S4), reporting an AUC value of 0.86 (95\%CI: $0.84-0.89$ ), with a sensitivity of 0.81 and a specificity of 0.87 (Table 2; Figure 2) in the training set. The performance was equivalent in the test cohort, with an AUC of 0.87 (95\%CI: 0.83-0.92), a sensitivity of 0.82 and a specificity of 0.86 (Table 2; Figure 2).

Table 2. AUC, sensitivity and specificity of the 3-transcript signature.

\begin{tabular}{cccccc}
\hline Study & Thresholds & Sensitivity & Specificity & AUC & 95\% CI \\
\hline GSE64456 & 10.80 & 0.87 & 0.90 & 0.93 & $0.89-0.96$ \\
\hline GSE72829 & 2.96 & 0.86 & 0.90 & 0.94 & $0.90-0.97$ \\
\hline GSE6269 & 12.49 & 1.00 & 0.75 & 0.84 & $0.66-1.00$ \\
\hline GSE20346 & 7.00 & 0.89 & 0.92 & 0.92 & $0.84-1.00$ \\
\hline GSE40012 & 7.07 & 0.82 & 0.75 & 0.83 & $0.75-0.91$ \\
\hline GSE40396 & 11.64 & 0.90 & 0.88 & 0.92 & $0.83-1.00$ \\
\hline GSE42026 & 8.27 & 1.00 & 0.94 & 0.95 & $0.90-1.00$ \\
\hline GSE25504 & 10.34 & 1.00 & 0.89 & 0.96 & $0.86-1.00$ \\
\hline GSE60244 & 9.75 & 0.72 & 0.95 & 0.90 & $0.84-0.96$ \\
\hline GSE63990 & 6.83 & 0.93 & 0.88 & 0.93 & $0.88-0.97$ \\
\hline GSE69529 & 792.62 & 0.75 & 0.65 & 0.76 & $0.69-0.82$ \\
\hline Training set & 439.56 & 0.81 & 0.87 & 0.86 & $0.84-0.89$ \\
\hline Test set & 439.77 & 0.82 & 0.86 & 0.87 & $0.83-0.92$ \\
\hline
\end{tabular}



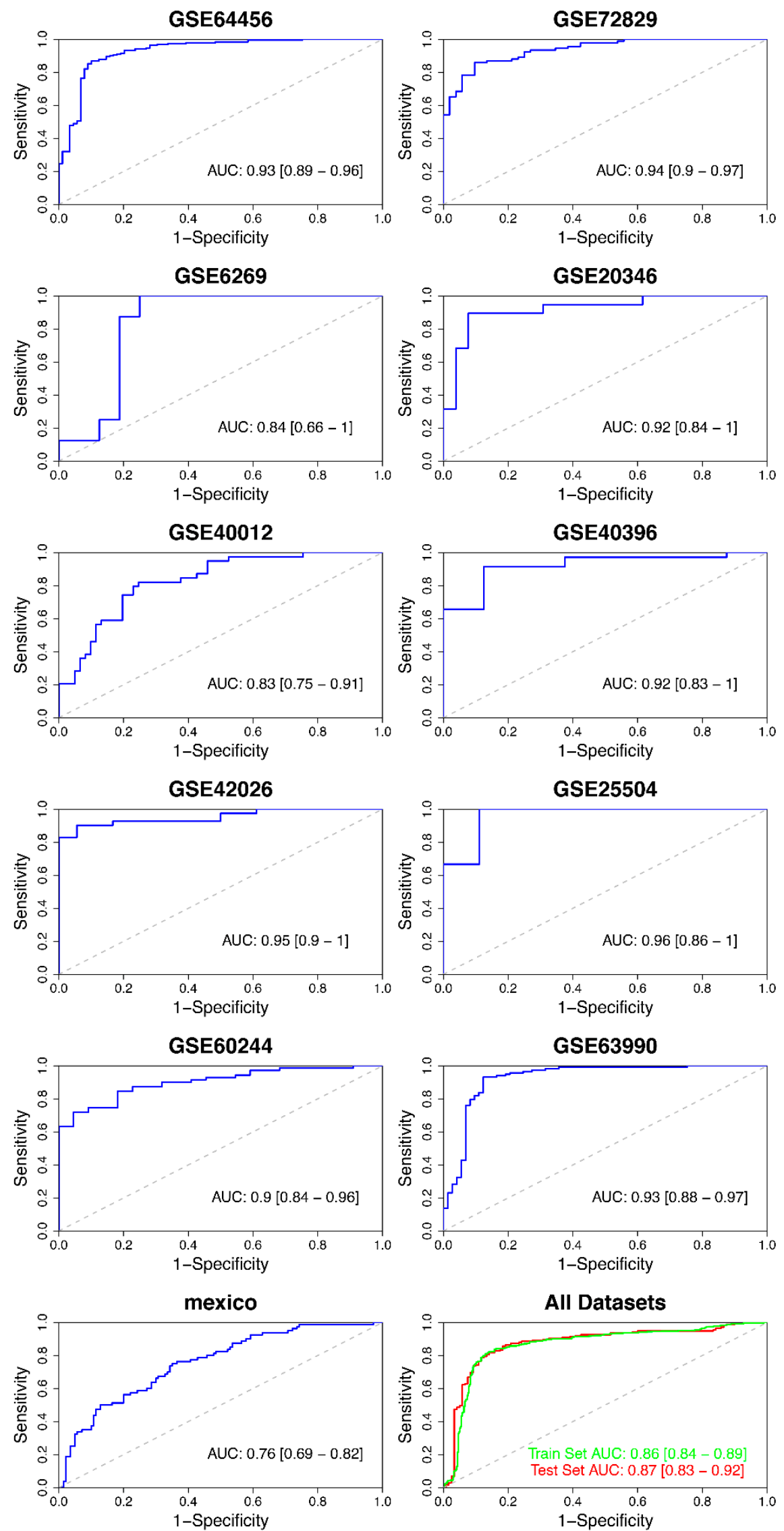

Figure 2. Evaluation of the 3-transcript signature performance in individual studies as well as in the training and test cohorts. AUC values and 95\% CI are provided. 
We further evaluated the performance of the 3-transcript model to differentiate viral from bacterial cases in each individual study; AUC values calculated ranged from 0.76 to 0.96 (Table 2, Figure 2). The lower value of AUC (AUC: 0.76 (95\%CI: 0.69-0.82); sensitivity: 0.75 and specificity: 0.65) was achieved in the Mexican cohort (RNA-seq data; GSE69529), and this low value probably reflects the heterogeneous nature of the cohort, which included patients affected by a mild disease.

\section{Discussion}

Both viral and bacterial infections occur with unspecific clinical symptoms, especially in early stages of the disease. In fact, viral and bacterial infections are often indistinguishable when considering only clinical settings and, therefore, empirical therapies are often administered as a preventive measure. The excessive use of antibiotics has led to an alarming increase in bacterial resistance and, in parallel, healthcare costs. The first step towards more precise antibiotic administration is the availability of faster, more sensitive, and accurate diagnostic tests. However, the tests currently available have several limitations; for instance, the gold standard of using bacterial cultures usually takes a long time to produce results. Although microbiological diagnosis has improved since the emergence of PCR-based assays, these tests do not always detect the causative pathogen, as available panels only interrogate the most frequent pathogens (requiring a priori suspicion of the pathogen), and sometimes they detect residual remains of a past infection.

In the present study, we conducted a multi-cohort meta-analysis using high-throughput (microarray and RNAseq) data available in public databases ( $n=1209$ samples) from blood transcriptomic studies including virus and bacteria-infected patients to find the best minimum gene expression signature that differentiates between both types of infections in all possible scenarios. Meta-analysis of transcriptomic data has proven to be a useful approach to discover gene expression signatures specific to different infectious diseases $[5,18,20]$, raising the statistical power compared with individual studies, and finding common trends in transcriptomic response under different conditions, pathogens, and demographic features. Using a gene signature candidate approach following a PReMS algorithm, we obtained a biosignature of 3-gene transcriptomics that accurately distinguishes viral from bacterial infections with high sensitivity and specificity. This signature also performed well when validated in all individual studies (Table 3; Figure 2), pointing to the functional versatility of the three-transcript signature in very different infection contexts. Two of the three genes in the signature, namely BAFT and ISG15, are both related to immune processes and, while the former is involved in several differentiation processes of some immune cells, the latter plays a key role in the immune response to RNA and DNA viruses [30-32]. On the other hand, the DNMT1 gene encodes for a protein that is responsible for maintaining DNA methylation patterns after replication and it has been shown that some viral [33,34] and bacterial [35] infections can induce the expression of this gene.

Table 3. Genes included in the viral vs bacterial 3-gene transcriptomic signature. LRC = logistic regression coefficient.

\begin{tabular}{ccc}
\hline Gene Symbol & Gene Name & LRC \\
\hline BATF & Basic Leucine Zipper & -1.16 \\
& ATF-Like Transcription Factor & 0.64 \\
\hline ISG15 & ISG15 Ubiquitin Like Modifier & 1.24 \\
\hline
\end{tabular}

Although knowledge of the functional features of these genes is of great interest, the most important issue in the context of biomarker discovery research is their capability to differentiate both types of infections, regardless of their role in the context of the pathophysiology of the disease. It occurs very often that candidate genes have unknown function, but this fact does not invalidate its potential to have specific diagnostic biomark- 
ers. For instance, Herberg et al. [4] discovered a two-transcript signature from microarray expression data, which discriminated between viral and bacterial infections with no known function of the genes involved. Despite this, the two-transcript signature was successfully tested and validated in prospective and other retrospective cohorts, and using different gene-expression technologies $[5,6,36]$. In the same line, two long non-coding RNAs have been recently proposed as biomarkers associated with viral infections, showing high performance capability in separating viral from healthy phenotypes [36]; their role, however, is completely unknown.

The main advantages of a 3-gene signature are its easy implementation in a diagnostic test, given the low number of genes needed, and its functionality under different conditions derived from the multi-cohort study. Even though RNA-seq and microarrays are emerging as the most powerful screening approaches to discover host RNA signatures related to infectious diseases, both have inherent problems such as a higher error rate than traditional Sanger sequencing, standardization, and reproducibility issues [10]. Therefore, before any biomarker is translated into a clinical test, it needs to be validated using well-standardized technologies [6] in proper clinical settings. Consequently, further effort is needed to validate the three-biomarker signature using robust molecular techniques such as real time-PCR (qPCR) [6] or nCounter (Nanostring ${ }^{\circledR}$ ) [10]. The qPCR is currently the "gold standard" in gene expression studies. Many studies have proven that qPCR is a suitable method to validate microarrays and RNA-seq findings, reporting a strong correlation between microarray and qPCR results [37]. Furthermore, qPCR-based assays are already widely used in hospital settings because this is a technique with high accuracy, which is also relatively cheap and fast [6]. However, establishing a detailed laboratory qPCR protocol that includes a careful selection of reference genes for each specific condition and good laboratory practices is crucial to successfully convert a host transcriptional signature into a qPCR assay that can be used in a diagnostics laboratory routinely [6].

Even though the development of a bedside test based on host transcriptomic biomarkers is highly desirable, this goal is not easy to achieve due to technical limitations. Nonetheless, this situation will most probably change soon thanks to new emergent technologies that will allow for sensitive and qualitative detection of gene expression within a short time frame. It is likely that in the next few years, we will see the application of the first host gene expression diagnostic tests for infectious diseases in clinical settings and, more importantly, an improvement in the diagnosis and treatment of infectious diseases [10].

\section{Conclusions}

Our results suggest that different infectious diseases are associated with different patterns of genes that turn on or off, constituting specific molecular signatures, which can be used to quickly identify viral or bacterial infections. We found three genes, namely $B A T F$, ISG15 and DNMT1, which can distinguish viral from bacterial infections in a wide range of cohorts including different pathogens, ages and populations, and with potential to become clinical biomarkers for infectious diseases in a clinical setting. As occurred in previous studies $[4-6,15,36]$, the role of biomarkers of infection is often unknown; this fact, however, does not diminish the importance of their capability to distinguish viral from bacterial infections. In our study, the concurrence of these biomarkers in a significant number of independent studies points to their important role in the process of infection, and this observation strongly suggests the need for further investigations.

The present study represents a step forward towards the use of host gene expression signatures in clinical settings. Due to the nature of our meta-analysis that uses retrospective data from 11 previously published studies, a validation cannot be done using the original samples. Therefore, further effort will be needed to collect new samples from viral and bacterial infected patients to further explore the 3-transcript signature in a new prospective cohort. Moreover, the translation of the selected transcriptomic biomarkers into a clinical test for diagnosis, prognosis or risk assessment needs further validation, as well as consideration of different scenarios, including illness severity, time points in the course of the 
infectious disease, parasitic infections, and other inflammatory diseases. In this context, a 3-transcript qPCR validation assay or alike (e.g., using the Nano String platform) might be also of interest before developing a point-of-care test.

There are still many challenges to overcome before host gene expression signatures can be introduced into a point-of-care molecular diagnostic test. However, signatures based on host gene expression biomarkers have a great potential for the diagnosis of infectious diseases; we envisage that their use in clinical diagnostic tests will skyrocket in the next few years.

\section{Material and Methods}

\subsection{Sample Groups}

We queried the public gene expression microarray repository Gene Expression Omnibus (GEO) for human gene expression datasets using the following terms: "viral" and/or "bacterial". We retained only those studies containing microarray expression or RNA-seq data from whole blood samples of virus- or bacteria-infected patients. Eleven studies $(n=1209$ samples) were included in the metanalysis (see details in Table 1): GSE64456 [19] $(n=279)$, GSE72829 [4] $(n=144)$, GSE6269 [22] $(n=24)$, GSE20346 [23] $(n=45), \mathrm{GSE} 40012$ [24] $(n=100), \mathrm{GSE} 40396$ [25] $(n=43), \mathrm{GSE} 42026$ [26] $(n=59), \mathrm{GSE} 25504$ [27] $(n=12), \operatorname{GSE60244~[28]~}(n=93), \operatorname{GSE69529}$ [21] $(n=220)$ and GSE63990 [29] $(n=190)$, including patients with bacterial and viral infections (Table S1).

\subsection{Data Processing and Statistical Analysis}

To merge and integrate the public viral vs. bacterial transcriptomic studies, we first normalized and pre-processed each dataset separately using the package Lumi [38] for Illumina ${ }^{\circledR}$ microarrays data and the package Oligo [39] for Affymetrix ${ }^{\circledR}$ datasets. RNA-seq data were pre-processed as described in [5].

We first merged these databases keeping only common genes included in all of them. Subsequently, we used the R package COCONUT (COmbat CO-Normalization Using conTrols) to combine all datasets into one and reduce batch effects in the meta-analysis [20]. After that, we only used for the follow-up analyses the candidate biomarkers reported in these studies as capable of differentiating between viral and bacterial infections. Only 64 out 163 candidate genes were present in all databases (Table S2) and, therefore, these 64 candidate genes were used as input to explore the minimum specific transcript signature for distinguishing viral from bacterial infection. We used PReMS [40] in a randomly split dataset removing healthy controls: training set $(n=914)$ and validation set $(n=295)$. PReMS investigates different logistic regression models built from optimal subsets of the candidate genes while increasing the model size iteratively. PReMS was the preferred method as it tends to choose signatures with a smaller number of genes without losing model accuracy, which would facilitate its future translation into a point-of-care test [10]. We tested first a model with a maximum of 15 genes and then explored how the predictive log-likelihood values change with the number of genes to find the signature with the minimum number of transcripts with optimum performance and facilitate its translation into the clinical routine.

Finally, the accuracy of the model estimated by PReMS was calculated as the AUC using the R package $p R O C$ [41] in both training and test cohorts as well as in all independent studies from the multi-cohort study. The Wilcoxon test was used to assess statistical significance between viral and bacterial groups. Functional pathways analysis was carried out through the Clusterprofiler [42] R package. We used the package enrichplot [43] for graphically displaying the results obtained. Heatmap representation of the top 14 genes from the optimal model was carried out with the ComplexHeatmap R package [44].

All analyses and graphical representations were conducted using $R$ software version 3.6.4 (www.r-project.org/, accessed on 26 January 2021). 
Supplementary Materials: The following are available online at https:/ / www.mdpi.com/1422-006 $7 / 22 / 6 / 3148 /$ s1.

Author Contributions: A.S., F.M.-T. and R.B.-A. conceived and designed the study. R.B.-A., A.S., X.B., J.P.-S. and A.G.-C. analyzed the data. A.S., R.B.-A., A.G.-C. and M.C.-L. wrote the first draft of the manuscript, which was revised by F.M.-T. All the authors contributed to the final version of the manuscript. All authors have read and agreed to the published version of the manuscript.

Funding: This study received support from projects: GePEM (Instituto de Salud Carlos III(ISCIII)/PI16/ 01478/Cofinanciado FEDER), DIAVIR (Instituto de Salud Carlos III(ISCIII)/DTS19/00049/Cofinanciado FEDER; Proyecto de Desarrollo Tecnológico en Salud), Resvi-Omics (Instituto de Salud Carlos III(ISCIII)/PI19/01039/Cofinanciado FEDER), BI-BACVIR (PRIS-3; Agencia de Conocimiento en Salud (ACIS) - Servicio Gallego de Salud (SERGAS) —Xunta de Galicia; Spain), Programa Traslaciona Covid-19 (ACIS-Servicio Gallego de Salud (SERGAS)—Xunta de Galicia; Spain) and Axencia Galega de Innovación (GAIN; IN607B 2020/08-Xunta de Galicia; Spain) awarded to A.S.; and projects ReSVinext (Instituto de Salud Carlos III(ISCIII)/PI16/01569/Cofinanciado FEDER), Enterogen (Instituto de Salud Carlos III(ISCIII)/ PI19/01090/Cofinanciado FEDER), and Axencia Galega de Innovación (GAIN; IN845D 2020/23—Xunta de Galicia; Spain) awarded to F.M.-T.

Institutional Review Board Statement: Not applicable.

Informed Consent Statement: Not applicable.

Data Availability Statement: Not applicable.

Acknowledgments: We gratefully acknowledge CESGA (Supercomputing Centre of Galicia, Santiago de Compostela, Spain) for computing availability, web hosting and support.

Conflicts of Interest: The authors declare no conflict of interest.

\section{References}

1. Martinón-Torres, F.; Salas, A.; Rivero-Calle, I.; Cebey-López, M.; Pardo-Seco, J.; Herberg, J.A.; Boeddha, N.P.; Klobassa, D.S.; Secka, F.; Paulus, S. Life-threatening infections in children in Europe (the EUCLIDS Project): A prospective cohort study. Lancet Child Adolesc. Health 2018, 2, 404-414. [CrossRef]

2. Hall, C.B.; Weinberg, G.A.; Iwane, M.K.; Blumkin, A.K.; Edwards, K.M.; Staat, M.A.; Auinger, P.; Griffin, M.R.; Poehling, K.A.; Erdman, D. The burden of respiratory syncytial virus infection in young children. N. Engl. J. Med. 2009, 360, 588-598. [CrossRef] [PubMed]

3. Le Doare, K.; Nichols, A.-L.; Payne, H.; Wells, R.; Navidnia, S.; Appleby, G.; Calton, E.; Sharland, M.; Ladhani, S.N.; Network, C. Very low rates of culture-confirmed invasive bacterial infections in a prospective 3-year population-based surveillance in Southwest London. Arch. Dis. Child. 2014, 99, 526-531. [CrossRef] [PubMed]

4. Herberg, J.A.; Kaforou, M.; Wright, V.J.; Shailes, H.; Eleftherohorinou, H.; Hoggart, C.J.; Cebey-López, M.; Carter, M.J.; Janes, V.A.; Gormley, S. Diagnostic test accuracy of a 2-transcript host RNA signature for discriminating bacterial vs viral infection in febrile children. JAMA 2016, 316, 835-845. [CrossRef]

5. Barral-Arca, R.; Pardo-Seco, J.; Martinon-Torres, F.; Salas, A. A 2-transcript host cell signature distinguishes viral from bacterial diarrhea and it is influenced by the severity of symptoms. Sci. Rep. 2018, 8, 1-7. [CrossRef] [PubMed]

6. Gómez-Carballa, A.; Cebey-López, M.; Pardo-Seco, J.; Barral-Arca, R.; Rivero-Calle, I.; Pischedda, S.; Currás-Tuala, M.J.; GómezRial, J.; Barros, F.; Martinón-Torres, F. A qPCR expression assay of IFI44L gene differentiates viral from bacterial infections in febrile children. Sci. Rep. 2019, 9, 1-12. [CrossRef] [PubMed]

7. Chu, D.K.; Pan, Y.; Cheng, S.; Hui, K.P.; Krishnan, P.; Liu, Y.; Ng, D.Y.; Wan, C.K.; Yang, P.; Wang, Q. Molecular diagnosis of a novel coronavirus (2019-nCoV) causing an outbreak of pneumonia. Clin. Chem. 2020, 66, 549-555. [CrossRef]

8. Mahony, J.B. Detection of respiratory viruses by molecular methods. Clin. Microbiol. Rev. 2008, 21, 716-747. [CrossRef]

9. Ramilo, O.; Mejías, A. Shifting the paradigm: Host gene signatures for diagnosis of infectious diseases. Cell Host Microbe 2009, 6, 199-200. [CrossRef]

10. Gliddon, H.D.; Herberg, J.A.; Levin, M.; Kaforou, M. Genome-wide host RNA signatures of infectious diseases: Discovery and clinical translation. Immunology 2018, 153, 171-178. [CrossRef]

11. Cebey-López, M.; Salas, A. Recognising the asymptomatic enemy. Lancet Infect. Dis. 2021, 21, 305-306. [CrossRef]

12. Mejias, A.; Dimo, B.; Suarez, N.M.; Garcia, C.; Suarez-Arrabal, M.C.; Jartti, T.; Blankenship, D.; Jordan-Villegas, A.; Ardura, M.I.; Xu, Z.; et al. Whole blood gene expression profiles to assess pathogenesis and disease severity in infants with respiratory syncytial virus infection. PLoS Med. 2013, 10, e1001549. [CrossRef] [PubMed]

13. Berry, M.P.; Graham, C.M.; McNab, F.W.; Xu, Z.; Bloch, S.A.; Oni, T.; Wilkinson, K.A.; Banchereau, R.; Skinner, J.; Wilkinson, R.J.; et al. An interferon-inducible neutrophil-driven blood transcriptional signature in human tuberculosis. Nature 2010, 466, 973-977. [CrossRef] [PubMed] 
14. Zak, D.E.; Penn-Nicholson, A.; Scriba, T.J.; Thompson, E.; Suliman, S.; Amon, L.M.; Mahomed, H.; Erasmus, M.; Whatney, W.; Hussey, G.D.; et al. A blood RNA signature for tuberculosis disease risk: A prospective cohort study. Lancet 2016, 387, $2312-2322$. [CrossRef]

15. Kaforou, M.; Herberg, J.A.; Wright, V.J.; Coin, L.J.M.; Levin, M. Diagnosis of bacterial infection using a 2-transcript host RNA signature in febrile infants 60 days or younger. JAMA 2017, 317, 1577-1578. [CrossRef] [PubMed]

16. Bhattacharya, S.; Rosenberg, A.F.; Peterson, D.R.; Grzesik, K.; Baran, A.M.; Ashton, J.M.; Gill, S.R.; Corbett, A.M.; Holden-Wiltse, J.; Topham, D.J.; et al. Transcriptomic biomarkers to discriminate bacterial from nonbacterial infection in adults hospitalized with respiratory illness. Sci. Rep. 2017, 7, 6548. [CrossRef]

17. Sampson, D.L.; Fox, B.A.; Yager, T.D.; Bhide, S.; Cermelli, S.; McHugh, L.C.; Seldon, T.A.; Brandon, R.A.; Sullivan, E.; Zimmerman, J.J.; et al. A Four-Biomarker Blood Signature Discriminates Systemic Inflammation Due to Viral Infection Versus Other Etiologies. Sci. Rep. 2017, 7, 2914. [CrossRef]

18. Barral-Arca, R.; Gómez-Carballa, A.; Cebey-López, M.; Bello, X.; Martinón-Torres, F.; Salas, A. A meta-analysis of multiple whole blood gene expression data unveils a diagnostic host-response transcript signature for respiratory syncytial virus. Int. J. Mol. Sci. 2020, 21, 1831. [CrossRef] [PubMed]

19. Mahajan, P.; Kuppermann, N.; Mejias, A.; Suarez, N.; Chaussabel, D.; Casper, T.C.; Smith, B.; Alpern, E.R.; Anders, J.; Atabaki, S.M.; et al. Association of RNA biosignatures with bacterial infections in febrile infants aged 60 days or younger. JAMA 2016, 316, 846-857. [CrossRef]

20. Sweeney, T.E.; Braviak, L.; Tato, C.M.; Khatri, P. Genome-wide expression for diagnosis of pulmonary tuberculosis: A multicohort analysis. Lancet. Respir. Med. 2016, 4, 213-224. [CrossRef]

21. DeBerg, H.A.; Zaidi, M.B.; Altman, M.C.; Khaenam, P.; Gersuk, V.H.; Campos, F.D.; Perez-Martinez, I.; Meza-Segura, M.; Chaussabel, D.; Banchereau, J. Shared and organism-specific host responses to childhood diarrheal diseases revealed by whole blood transcript profiling. PLoS ONE 2018, 13, e0192082. [CrossRef]

22. Ramilo, O.; Allman, W.; Chung, W.; Mejias, A.; Ardura, M.; Glaser, C.; Wittkowski, K.M.; Piqueras, B.; Banchereau, J.; Palucka, A.K. Gene expression patterns in blood leukocytes discriminate patients with acute infections. Blood 2007, 109, 2066-2077. [CrossRef] [PubMed]

23. Parnell, G.; McLean, A.; Booth, D.; Huang, S.; Nalos, M.; Tang, B. Aberrant cell cycle and apoptotic changes characterise severe influenza A infection-a meta-analysis of genomic signatures in circulating leukocytes. PLoS ONE 2011, 6, e17186. [CrossRef] [PubMed]

24. Parnell, G.P.; McLean, A.S.; Booth, D.R.; Armstrong, N.J.; Nalos, M.; Huang, S.J.; Manak, J.; Tang, W.; Tam, O.-Y.; Chan, S. A distinct influenza infection signature in the blood transcriptome of patients with severe community-acquired pneumonia. Crit. Care 2012, 16, R157. [CrossRef]

25. $\mathrm{Hu}, \mathrm{X} . ; \mathrm{Yu}, \mathrm{J}$.; Crosby, S.D.; Storch, G.A. Gene expression profiles in febrile children with defined viral and bacterial infection. Proc. Natl. Acad. Sci. USA 2013, 110, 12792-12797. [CrossRef] [PubMed]

26. Herberg, J.A.; Kaforou, M.; Gormley, S.; Sumner, E.R.; Patel, S.; Jones, K.D.; Paulus, S.; Fink, C.; Martinon-Torres, F.; Montana, G. Transcriptomic profiling in childhood H1N1/09 influenza reveals reduced expression of protein synthesis genes. J. Infect. Dis. 2013, 208, 1664-1668. [CrossRef] [PubMed]

27. Dickinson, P.; Smith, C.L.; Forster, T.; Craigon, M.; Ross, A.J.; Khondoker, M.R.; Ivens, A.; Lynn, D.J.; Orme, J.; Jackson, A. Whole blood gene expression profiling of neonates with confirmed bacterial sepsis. Genom. Data 2015, 3, 41-48. [CrossRef] [PubMed]

28. Suarez, N.M.; Bunsow, E.; Falsey, A.R.; Walsh, E.E.; Mejias, A.; Ramilo, O. Superiority of transcriptional profiling over procalcitonin for distinguishing bacterial from viral lower respiratory tract infections in hospitalized adults. J. Infect. Dis. 2015, 212, 213-222. [CrossRef]

29. Tsalik, E.L.; Henao, R.; Nichols, M.; Burke, T.; Ko, E.R.; McClain, M.T.; Hudson, L.L.; Mazur, A.; Freeman, D.H.; Veldman, T. Host gene expression classifiers diagnose acute respiratory illness etiology. Sci. Transl. Med. 2016, 8, ra311-ra322. [CrossRef]

30. Hsiang, T.Y.; Zhao, C.; Krug, R.M. Interferon-induced ISG15 conjugation inhibits influenza A virus gene expression and replication in human cells. J. Virol. 2009, 83, 5971-5977. [CrossRef]

31. Kuang, Z.; Seo, E.J.; Leis, J. Mechanism of inhibition of retrovirus release from cells by interferon-induced gene ISG15. J. Virol. 2011, 85, 7153-7161. [CrossRef] [PubMed]

32. Okumura, A.; Pitha, P.M.; Harty, R.N. ISG15 inhibits Ebola VP40 VLP budding in an L-domain-dependent manner by blocking Nedd4 ligase activity. Proc. Natl. Acad. Sci. USA 2008, 105, 3974-3979. [CrossRef] [PubMed]

33. Vivekanandan, P.; Daniel, H.D.; Kannangai, R.; Martinez-Murillo, F.; Torbenson, M. Hepatitis B virus replication induces methylation of both host and viral DNA. J. Virol. 2010, 84, 4321-4329. [CrossRef]

34. Laurson, J.; Khan, S.; Chung, R.; Cross, K.; Raj, K. Epigenetic repression of E-cadherin by human papillomavirus 16 E7 protein. Carcinogenesis 2010, 31, 918-926. [CrossRef] [PubMed]

35. Tolg, C.; Sabha, N.; Cortese, R.; Panchal, T.; Ahsan, A.; Soliman, A.; Aitken, K.J.; Petronis, A.; Bagli, D.J. Uropathogenic E. coli infection provokes epigenetic downregulation of CDKN2A (p16INK4A) in uroepithelial cells. Lab. Investig. 2011, 91, 825-836. [CrossRef] [PubMed]

36. Barral-Arca, R.; Gomez-Carballa, A.; Cebey-Lopez, M.; Curras-Tuala, M.J.; Pischedda, S.; Viz-Lasheras, S.; Bello, X.; MartinonTorres, F.; Salas, A. RNA-Seq Data-Mining Allows the Discovery of Two Long Non-Coding RNA Biomarkers of Viral Infection in Humans. Int. J. Mol. Sci. 2020, 21, 2748. [CrossRef] 
37. Morey, J.S.; Ryan, J.C.; Van Dolah, F.M. Microarray validation: Factors influencing correlation between oligonucleotide microarrays and real-time PCR. Biol. Proced. Online 2006, 8, 175-193. [CrossRef] [PubMed]

38. Du, P.; Kibbe, W.A.; Lin, S.M. lumi: A pipeline for processing Illumina microarray. Bioinformatics 2008, 24, 1547-1548. [CrossRef]

39. Bumgarner, R. Overview of DNA microarrays: Types, applications, and their future. Curr. Protoc. Mol. Biol. 2013, 101. [CrossRef]

40. Hoggart, C.J. PRReMS: Parallel Regularised Regression Model Search for bio-signature discovery. bioRxiv 2018, 355479. [CrossRef]

41. Robin, X.; Turck, N.; Hainard, A.; Tiberti, N.; Lisacek, F.; Sanchez, J.-C.; Müller, M. pROC: An open-source package for R and S+ to analyze and compare ROC curves. Bmc Bioinform. 2011, 12, 77. [CrossRef] [PubMed]

42. Yu, G.; Wang, L.G.; Han, Y.; He, Q.Y. clusterProfiler: An R package for comparing biological themes among gene clusters. Omics J. Integr. Biol. 2012, 16, 284-287. [CrossRef] [PubMed]

43. Yu, G. enrichplot: Visualization of Functional Enrichment Result; R Package Version 1.6.1. 2019. Available online: https://rdrr.io/ bioc/enrichplot/ (accessed on 11 March 2021).

44. Gu, Z.; Eils, R.; Schlesner, M. Complex heatmaps reveal patterns and correlations in multidimensional genomic data. Bioinformatics 2016, 32, 2847-2849. [CrossRef] [PubMed] 